\title{
Bridging the Gap: Induced Pluripotent Stem Cell Derived Endothelial Cells for 3D Vascular Assembly
}

\author{
Bria L Macklin ${ }^{1}$ and Sharon Gerecht ${ }^{1,2}$
}

${ }^{1}$ Department of Chemical and Biomolecular Engineering, Institute for NanoBioTechnology, Johns Hopkins University, Baltimore, MD 21218

${ }^{2}$ Department of Materials Science and Engineering, Johns Hopkins University, Baltimore, MD 21218 gerecht@jhu.edu 
Key Words: Vascular Assembly, iPSC, endothelial, Three-Dimensional

Abstract: As cardiovascular disease continues to take the lives of so many patients, it is paramount that scientists and engineers construct reliable, lasting vascular therapies. Stem cell therapies for cardiovascular disease have the potential to provide patient specific treatments in order to heal or replace the diseased vasculature. This review highlights recent advances in endothelial cell (EC) differentiation from human pluripotent stem cells (hPSCs). We then describe in vitro studies focusing on understanding three-dimensional vascular assembly using human primary ECs and biomaterials developed for these systems. Finally, we highlight the unprecedented opportunity that hPSC- derived ECs provide to study human vascular development for fundamental understanding and toward the development of therapeutics.

\section{Introduction}

\section{Need and advantages for vascular therapies}

In the developing embryo, the cardiovascular system is one of the first organ systems to develop, due to critical necessity for supply of oxygen and nutrients [1]. The cardiovascular system remains just as essential in human adults. As a consequence, any damage or disease effecting the vasculature or heart often results in diminished capacity or death. In fact, diseases of the vasculature continue to be the leading cause of death in the US. It is estimated that about 2,200 Americans die of cardiovascular disease every day[2].

The need for long-term solutions is more dire than ever. Current therapies utilize stents and grafts which only provide a short term solution. Vascular therapies utilizing cell-free constructs to rebuild the damaged vessels that harness the body's own regenerative capacity, are an ideal solution to treat cardiovascular diseases. However, these may have limited therapeutic potential to severely damaged vasculature in cases of systematic or chronic diseases. Engineered cell based therapies are an excellent alternative approach with implications for long-term treatment clinically. Forming vascular networks in three-dimensional (3D) hydrogels would allow us to create disease free vessels that can be implanted in vivo to recover the vasculature. These engineered constructs also provide researchers the ability to closely study vascular assembly.

\section{Vasculogenesis and angiogenesis}

The development of functional vasculature is vital to the survival of a growing embryo. These early networks serve as a transport system for both nutrients and waste. Vasculogenesis followed by angiogenesis are the two primary processes that regulate vascular assembly in the embryo and human adults, respectively [1,3] Briefly, vasculogenesis is the formation of vessels from a primary vascular plexus; Angiogenesis is formation of vessels from pre-existing vasculature. This review will focus on in vitro tube assembly via vasculogenesis continuing onto angiogenesis.

\section{Cell sources for vascular therapy}

\section{Adult Stem and Mature Cells}

The most obvious choice of cell types to repair damaged vasculature is the patient's own somatic cells. However, these cells can retain the symptoms that lead to cardiovascular disease, including oxidative stress, vascular inflammation, and endothelial dysfunction[4]. Endothelial progenitor cells (EPCs), another logical source, are cells that circulate in the blood and are recruited to sites of vascular injury to participate in new vessel formation [5]. EPCs have been proven effective in clinical transplantation trials for varying cardiovascular diseases; please see details of the studies in [6]. Unfortunately, it has been suggested in previous animal studies that about $0.5-2.0 \times 10^{4}$ human EPCs/g body weight would be required to achieve adequate results in hind limb ischemia [6]. Treating 
a human ischemic patient would require about 12 liters of blood. In addition, cardiovascular disease and many of the associated diseases reduce the number of circulating EPCs [6], making these cells virtually unattainable for vascular therapy.

\section{Human pluripotent stem cells a constant, renewable cell source}

Human pluripotent stem (hPSCs) cells have become popular in the field of tissue engineering as a source to derive a multitude of cell types [7-10]. These cells have the unique advantage of being able to differentiate into cells of all three germ layers. Human PSCs can be isolated from the inner cell mass of a blastocyst, termed embryonic stem cells, and can be expanded in vitro indefinitely. A more recent source for hPSCs are induced pluripotent stem cells (iPSCs). Human iPSCs offer the opportunity for patient specific stem cell derived therapies. Somatic or adult stem cells are taken from a patient and reprogrammed to express vital pluripotency characteristics by various means ranging from gene insertion to small molecule reprogramming [11, 12]. iPSCs can provide an inexhaustive, constant source of endothelial cells (ECs) to be utilized in vascular therapies.

\section{Pluripotent Stem Cell Derived Endothelial Cells}

A number of studies have demonstrated the differentiation of hPSCs into ECs[13-24]. Several different differentiation approaches have been utilized, all novel in their methodology but with a similar end goal: acquiring functional ECs.

\section{Differentiation Schemes}

While some groups opted to use embryoid body formation to induce hPSC differentiation[15, 21], others have demonstrated analogous differentiation using directed differentiation on 2D monolayers $[13,18,20,25]$. Despite the fact the differentiation kinetics differ in these approaches, it is important to note that in both approaches, the cell density plays a major role in the differentiation efficiency [26, 27]

Growth factors and other small molecules are a key factor in driving hPSCs into determined fates. The addition of Vascular Endothelial Growth Factor (VEGF) is a shared step among many differentiation methods, for inducing a vascular phenotype in vitro [13,15, 16, 19-22]. Sriram et al demonstrated that while VEGF alone can be used to direct vascular differentiation, VEGF supplemented with bone morphogenetic protein 4 (BMP-4) can be used to produce a larger population of $\mathrm{CD} 34^{+} \mathrm{CD} 31^{+} \mathrm{VEGFR2}{ }^{+}$cells from hPSCs. This group also utilized the small molecule $\mathrm{CHIR99021}$, a glycogen synthase kinase-3 $\beta$ inhibitor shown to induce mesoderm differentiation with $100 \%$ efficiency[28]. Patsch et al and Orlova et al also utilized CHIR99021 to induce mesoderm differentiation[16, 24]. SB431542, a transforming growth factor $\beta$ inhibitor is another widely used small molecule to guide vascular differentiation $[13,16,19,21,22]$.Differences in overall differentiation time can be correlated to growth factor and small molecule addition. In differentiation methods where CHIR99021 is used, the mesoderm inducing step ranges between $48-72$ hours $[13,16,24]$ as compared to other protocols where this step can take as much as 144 hours [15, 20, 22, 23].

In addition to biochemical cues, physical cues are suggested to regulate endothelial fate. We have previously shown that differentiation in low oxygen conditions, ie hypoxia, results in increased endothelial cell commitment [29]. Other cues such as matrix stiffness have been shown to induce mesodermal fate [30] and thus may potentially impact endothelial fate decisions.

In order to procure a pure population of ECs it is necessary to selectively sort out cells that highly express typical endothelial surface markers. This can be done using either Magnetic Activated Cells Sorting (MACS) or Fluorescent Activated Cell Sorting (FACS), both methods utilize a primary antibody stain. James et al utilized both MACS and FACS to sort out a CD31+ population[21]. Other positive surface markers that can be utilized for sorting are Vascular Endothelial-cadherin (VECad), CD34, Kinase Insert Domain Receptor (KDR)/Vascular endothelial growth factor receptor 2 (VEGFR2), and Neuropilin 1 (NRP-1) [13, 15, 18-20, 22, 24]. Another purification approach utilized by Lippman et al, involves seeding differentiated cells on tissue culture plates coated with a mixture of collagen IV and fibronectin and only culturing the cells that adhere to the coating. This method utilizes 
the affinity of ECs to bind to collagen coating surfaces, thus selecting against non-binding cells that are not true ECs [23].

Some groups opt to use multiple markers in order to obtain a very specific population of ECs [25, 31]. Prasain et al isolate a population of NRP $-1^{+} \mathrm{CD} 31^{+} \mathrm{CD} 144^{+}$cells. These cells are highly proliferative and yield an iPSC:EC ratio of $1: 1 \times 10^{8}$, in addition to being functional for up to 18 passages[25]. Just as vital as the purification method is in isolating a pure population of differentiated ECs, the conditions for their expansion are important too. Human PSC-ECs have been shown to be unstable, differentiating into other cells types and/or de-differentiating back to EC precursors, when cultured over time [21, 25]. James et al demonstrated that this can be inhibited for at least up to 20 days with the addition of SB431542 in both the differentiation and expansion steps[21].

\section{Network Assembly}

As briefly described before vasculogenesis is the de novo formation of vascular structures, whilst in angiogenesis vessels sprout from pre-existing vessels. De novo vascular assembly occurs in three steps, all initiating from cells of the mesoderm, one of the three embryonic germ layers. Beginning with the differentiation of blood islands from mesodermal cells. Blood islands are an early precursor to ECs, cells that comprise the inner most lining of blood vessels. Blood islands are multicellular masses of both angioblasts and hemapoietic cells. Next, angioblasts begin to differentiate into ECs, coinciding with an increase of the growth factors VEGFR2, VEGFR1, and tie-1, in addition to the extracellular matrix (ECM) proteins fibronectin and lamanin [1]. These ECs then give rise to the first vascular construct referred to as the primary vascular plexus. It is from this plexus that all other vessels will form via angiogenesis[3]. Angiogenesis begins with the activation of the tip cell, a specific EC that leads the sprouting process. This tip cell senses cues in the environment in order to guide the process. Stalk cells, which are directly interacting with the tip cell, begin to proliferate and form lumen in order to shape the newly formed vessels[32]. Stabilizing cells, pericytes are recruited newly formed tubes by EC derived ligands heparin-binding epidermal growth factor (HB-EGF) and platelet derived growth factor (PDGF)-BB[33].

In vitro vascular models are engineered to mimic the complex natural processes that occur in vivo. In order to recapitulate these events, all experimental parameters must be carefully chosen to achieve optimal network assembly in 3D matrix. These in vitro systems also offer researchers a tunable platform to study the events governing human vasculogenesis and angiogenesis to engineer better vascular therapies.

\section{Network Formation: Growth Factors and Small Molecules}

Indeed, in vitro systems for EC based vascular networks enabled researchers to study the macro and micro scale events of human vascular assembly. Many groups have identified the importance of specific growth factors, small molecules, and signaling pathways that regulate vessel formation in vitro. Utilizing human umbilical vein endothelial cells( HUVECs) in a 3D collagen matrix, Norden et al demonstrated the imperative role of GTPases Cdc42 and Rac1 on lumen formation, a process necessary for tube formation [34].

While innate growth factors secreted by ECs undergoing angiogenesis play a large role in vessel formation, Sacharidou et al has showed that when supplemented with several growth factors including stem cell factor, interleukin-3, stromal derived factor-1 $\alpha$, fibroblast growth factor-2 and insulin vascular network formation is increased. The group also found that by supplementing the growing networks with VEGF, the growth factor does not actively control the development of the vasculature. Rather, it acts as a priming agent to allow the cells to respond the the cocktail of growth factors mentioned above, resulting in increased network formation.

Analogous to angiogenesis in vivo, in vitro angiogenesis involves the secretion of ECM proteins and proteases to help the sprouting ECs degrade and remodel the matrix [35]. Fibronectin is a large glycoprotein that binds to many different ECM components, including collagen I, collagen IV, lamanin, 
and fibrin. In the absence of fibronectin, it has been shown that vascular assembly and ECs migration fails to occur, demonstrating its necessity for in vitro vascular assembly [36]. The addition of lamanin in 3D collagen based vascular models has been shown to regulate VEGF intake resulting in a higher population of KDR/VEGFR2 positive cells, in addition to promoting EC network formation [37]. Subsequently, the composition and structure of the matrix play a central role in the network kinetics, and will be discussed in details later within the review.

\section{Network Sustainability: Supporting Cells}

While 3D networks made of ECs provide insight into early vasculogenic and angiogenic events, models that utilize cocultures including mural and/or stromal supporting cells, offer more clinically relevant systems. Mural cells, which consist of vascular smooth muscle cells, pericytes and stromal cells, often fibroblasts, use both chemical and physical cues to stabilize and support the vasculature. Fibroblasts are the most abundant cells of connective tissue in animals, the cells secrete the ECM protein collagen. Fibroblasts also secrete the pro-angiogenic factors VEGF, PDGF and transforming growth factor- $\beta$ (TGF- $\beta$ ). These factors help to stabilize EC networks as well as promoting lumen formation[38]. Due to these properties, fibroblast have been used in Coculture studies of 3D vasculogenesis[39-41] Unlike fibroblasts, pericytes are a supporting cell type that directly interact with EC networks (Figure 1). Pericytes are directly imbedded into the basement membrane of EC lined tubes, and are present in tissue specific ratios on most microvessels. EC-pericyte interactions are regulated by a number of proteins that govern cell recruitment, proliferation, differentiation, and maturation of each cell type [42]. Several studies have focused on understanding EC-pericyte interactions during network formation demonstrating communication between the two cell type to recruit pericytes to adhere to the nascent EC network [33, 34, 43]. Vascular smooth muscle cells are present around larger vascular structures, secreting elastic ECM (Figure 1). This muscular layer allow for contractility of the vasculature, regulating key processes such as blood pressure[24]. vSMCs also have the ability to secrete proangiogenic factors in the presence of endothelial dysfunction[44].

\section{Biomaterials for Vascular Network Assembly}

As previously mentioned, the ECM provides the matrix for 3D vascular assembly. As such, 3D biomaterials are an imperative part of in vitro approach for vascular assembly in 3D. Sprouting vascular networks are constantly remodeling the ECM by way of traction forces, proteolytic activity (governed by matrix metalloproteinases), and cell-matrix adhesions [35]. An optimal biomaterial for vascular assembly must not only be biocompatible, but possess the optimal stiffness, density, and permeability to allow for vascular sprouting and growth to occur. Hydrogels, 3D crosslinked polymeric networks, can be designed to mimic the native cellular environment, using natural and synthetic polymer building blocks.

\section{Natural Hydrogels}

Hydrogels comprised of natural polymers, such as collagen, fibrin, and elastin, have a select advantage over synthetic materials in that their biocompatibility, biodegradability, and cell adhesion sites are inherent. Because of this, they are an exemplar choice for in vitro vascular models. While Matrigel matrix, a solubilized basement membrane extracted from mouse sarcoma cells, uses to examine cord-like formation from ECs, it is utilized as a 2D surface in vitro and not as a matrix for 3D vascular assembly. Type I collagen gels are a popular natural material utilized to host vascular networks[34, 37]. Integrin binding of $\alpha 2 \beta 1$ allows for EC sprouting and migration in collagen gel, making it a model material. It has been shown that when coculutred with pericytes, EC tubulogensis is less reliant on the integrin $\alpha 2 \beta 1$ as matrix remodeling increases. Instead integrins $\alpha 5 \beta 1, \alpha 3 \beta 1$, $\alpha 6 \beta 1$, and $\alpha 1 \beta 1$ interact with the large amount of ECM secreted by the EC and supporting mural cell [45]. Fibrin, a natural material typically involved in blood clotting, is another biomaterial hydrogel commonly used to encapsulate ECs[39]. Fibrin, like collagen, is conducive to vasculogenesis and angiogenesis due to integrin binding, specifically $\alpha 5 \beta 1$ and $\alpha v \beta 3$. 


\section{Semi-Synthetic Hydrogels}

Semi-synthetic hydrogels are an emerging new class of hydrogels that combine the biocompatibility of natural polymers with the customizability of synthetic materials. In the study of vasculature, semisynthetic hydrogels include mainly dextran and hyaluronic acid (HA) hydrogels. Acrylated hyaluronic acid (AHA) hydrogels further modified with adhesion motives and degradable by matrix metalloproteinases, have been shown to support network formation and sustainable [46] vascular structures (Figure 2A). These gels also have been utilized to display how both oxygen tension and matrix stiffness can regulate angiogenic sprouting[47]. Dextran hydrogels can also facilitate in vitro vascular sprouting and network formation. We recently utilized a dextran-gelatin hybrid hydrogel, to decouple the effect of mechanical properties and hypoxic conditions ([48]; (Figure 2B).

\section{Synthetic Hydrogels}

Synthetic hydrogels, including the commonly used Poly (Ethylene Glycol) (PEG), are highly tunable, in that their stiffness, degradability, ultrastructure, and crosslinking ability can be customized in the laboratory[49]. This customization allows for more predictable network formation leading to a more defined system. This advantage is particularly important as these therapies move to the clinical realm, as natural materials can have batch to batch differences and be rejected by host tissues. Peters et al has recently demonstrated how (PEG) hydrogels can be functionalized in order to permit formation of microvessels by EPCs. Similar to AHA hydrogels, PEG hydrogels were functionalized with adhesion motives and MMP cleavable peptide sequences to allow for degradation. These gels were able to form EC only networks for 30 days as well as recruit vSMCs when co-cultured in vitro [50]. Nguyen et a/ utilized a PEG based hydrogel functionalized with norbornene in order to study EC attachment and the effects of gel modulus on network formation. They found that at a medium modulus $(3100 \pm 220$ $\mathrm{Pa}$ ), the modified PEG gels permits longer capillary-like structures when compared with gels of a lower and higher modulus. However, this effect could be reversed by inhibiting VEGFR2/KDR signaling [51].

\section{Network Assembly of hPSC-ECs.}

While many different differentiation schemes have been developed, most studies utilize 2D matrigel cord formation assays to assess functionality in vitro. Some groups have opted to jump from 2D studies directly to in vivo examination, to demonstrate the functionality of the hPSC-ECs [18, 20, 24, 25]

There is a critical need to understand how these newly developed ECs assemble into functional network. Yet, most of the recent studies focusing on understanding human vascular assembly utilize mature ECs. These groups have led the way in understanding vascular kinetics and the engineering of vascular based therapies. The same detailed exploration and characterization of vascular assembly should also be employed using hPSCs-derived ECs. Such studies will not only provide insight toward human vascular development but are necessary and a critical step that must be taken in order for hPSC based cardiovascular disease solutions to become more clinically appealing.

Although not many, a small fraction of groups have investigated hPSC derived ECs in vitro[15, 19, 22, 40, 52]. Our lab has shown early vascular cells (EVCs), a term used to describe an early population of ECs and pericytes, forming network structures in both AHA hydrogels and a gelatin-ferulic acid based hypoxic hydrogels $[19,22]$. Network formation of iPSC-ECs in a 3D collagen gel is shown in Figure 3. Chan et al demonstrated the potential of iPSCs-EC in creating healthy vascular constructs for patients with diabetes. Taking hiPSCs reprogramed from somatic cells of a patient with type 1 diabetes, a disease which puts patients at a higher risk for developing cardiovascular disease, we 
showed that these cells can both differentiate into ECs as well as form networks in hydrogels, just as healthy hiPSCs can. Although much work has been done in mature cell lines, it is imperative that hPSC-ECs are analyzed in details for kinetic of 3D vascular assembly (Figure 4), and their ability to recruit supporting stromal and mural cells towards network longevity. Overall, ECs derived from hiPSCs can provide patients with personalized therapies in order to help combat the large toll cardiovascular disease is taking on the United States. A fully functional and characterized differentiation and maturation scheme as well their impact on surrounding tissue is necessary to ensure that these remedies will meet FDA standards. Such research will enable hiPSC-ECs to advance towards utilization in cell-based therapeutics.

\section{Acknowledgments}

Funding for our research reviewed in this manuscript include and the American Heart Association grant 15EIA22530000, National Science Foundation grant 1054415 and Maryland Stem Cell Research Fund (to S.G). 


\section{References}

1. Risau, W. and I. Flamme, Vasculogenesis. Annu Rev Cell Dev Biol, 1995. 11: p. 73-91.

2. Mozaffarian, D., et al., Executive Summary: Heart Disease and Stroke Statistics--2016 Update: A Report From the American Heart Association. Circulation, 2016. 133(4): p. 447-54.

3. Risau, W., Mechanisms of angiogenesis. Nature, 1997. 386(6626): p. 671-4.

4. Siti, H.N., Y. Kamisah, and J. Kamsiah, The role of oxidative stress, antioxidants and vascular inflammation in cardiovascular disease (a review). Vascul Pharmacol, 2015. 71: p. 40-56.

5. Yoder, M.C., Human endothelial progenitor cells. Cold Spring Harb Perspect Med, 2012. 2(7).

6. Ii, M., et al., Vascular Regeneration Therapy: Endothelial Progenitor Cells for Ischemic Diseases, in Regenerative Medicine - from Protocol to Patient: 5. Regenerative Therapies II, G. Steinhoff, Editor. 2016, Springer International Publishing: Cham. p. 35-57.

7. Burridge, P.W., et al., Chemically defined generation of human cardiomyocytes. Nat Methods, 2014. 11(8): p. 855-60.

8. Kusuma, S. and S. Gerecht, Derivation of Endothelial Cells and Pericytes from Human Pluripotent Stem Cells. Methods Mol Biol, 2016. 1307: p. 213-22.

9. Zhu, S., et al., Human pancreatic beta-like cells converted from fibroblasts. Nat Commun, 2016. 7: p. 10080.

10. Wen, Z., et al., Synaptic dysregulation in a human iPS cell model of mental disorders. Nature, 2014. 515(7527): p. 414-8.

11. Rony, I.K., et al., Inducing pluripotency in vitro: recent advances and highlights in induced pluripotent stem cells generation and pluripotency reprogramming. Cell Prolif, 2015. 48(2): p. 140-56.

12. Hou, P., et al., Pluripotent stem cells induced from mouse somatic cells by small-molecule compounds. Science, 2013. 341(6146): p. 651-4.

13. Sriram, G., et al., Efficient differentiation of human embryonic stem cells to arterial and venous endothelial cells under feeder- and serum-free conditions. Stem Cell Res Ther, 2015. 6: p. 261.

14. Reed, D.M., et al., Morphology and vasoactive hormone profiles from endothelial cells derived from stem cells of different sources. Biochem Biophys Res Commun, 2014. 455(3-4): p. 172-7.

15. Park, T.S., et al., Vascular progenitors from cord blood-derived induced pluripotent stem cells possess augmented capacity for regenerating ischemic retinal vasculature. Circulation, 2014. 129(3): p. 359-72.

16. Orlova, V.V., et al., Generation, expansion and functional analysis of endothelial cells and pericytes derived from human pluripotent stem cells. Nat Protoc, 2014. 9(6): p. 1514-31.

17. Schukur, L., et al., Directed differentiation of size-controlled embryoid bodies towards endothelial and cardiac lineages in RGD-modified poly(ethylene glycol) hydrogels. Adv Healthc Mater, 2013. 2(1): p. 195-205.

18. Samuel, R., et al., Generation of functionally competent and durable engineered blood vessels from human induced pluripotent stem cells. Proc Natl Acad Sci U S A, 2013. 110(31): p. 12774-9.

19. Kusuma, S., et al., Self-organized vascular networks from human pluripotent stem cells in a synthetic matrix. Proc Natl Acad Sci U S A, 2013. 110(31): p. 12601-6.

20. Tatsumi, R., et al., Simple and highly efficient method for production of endothelial cells from human embryonic stem cells. Cell Transplant, 2011. 20(9): p. 1423-30.

21. James, D., et al., Expansion and maintenance of human embryonic stem cell-derived endothelial cells by TGFbeta inhibition is Id1 dependent. Nat Biotechnol, 2010. 28(2): p. 161-6.

22. Chan, X.Y., et al., Three-Dimensional Vascular Network Assembly From Diabetic PatientDerived Induced Pluripotent Stem Cells. Arterioscler Thromb Vasc Biol, 2015. 35(12): p. 267785.

23. Lippmann, E.S., et al., Derivation of blood-brain barrier endothelial cells from human pluripotent stem cells. Nat Biotechnol, 2012. 30(8): p. 783-91. 
24. Patsch, C., et al., Generation of vascular endothelial and smooth muscle cells from human pluripotent stem cells. Nat Cell Biol, 2015. 17(8): p. 994-1003.

25. Prasain, N., et al., Differentiation of human pluripotent stem cells to cells similar to cord-blood endothelial colony-forming cells. Nat Biotechnol, 2014. 32(11): p. 1151-7.

26. Moon, S.H., et al., Optimizing human embryonic stem cells differentiation efficiency by screening size-tunable homogenous embryoid bodies. Biomaterials, 2014. 35(23): p. 5987-97.

27. Wilson, H.K., et al., Exploring the effects of cell seeding density on the differentiation of human pluripotent stem cells to brain microvascular endothelial cells. Fluids Barriers CNS, 2015. 12: p. 13.

28. Lam, A.Q., et al., Rapid and efficient differentiation of human pluripotent stem cells into intermediate mesoderm that forms tubules expressing kidney proximal tubular markers. J Am Soc Nephrol, 2014. 25(6): p. 1211-25.

29. Kusuma, S., et al., Low oxygen tension enhances endothelial fate of human pluripotent stem cells. Arterioscler Thromb Vasc Biol, 2014. 34(4): p. 913-20.

30. Przybyla, L., J.N. Lakins, and V.M. Weaver, Tissue Mechanics Orchestrate Wnt-Dependent Human Embryonic Stem Cell Differentiation. Cell Stem Cell, 2016. 19(4): p. 462-475.

31. Sahara, M., et al., Manipulation of a VEGF-Notch signaling circuit drives formation of functional vascular endothelial progenitors from human pluripotent stem cells. Cell Res, 2014. 24(7): p. 820-41.

32. Geudens, I. and H. Gerhardt, Coordinating cell behaviour during blood vessel formation. Development, 2011. 138(21): p. 4569-83.

33. Stratman, A.N., et al., Endothelial-derived PDGF-BB and HB-EGF coordinately regulate pericyte recruitment during vasculogenic tube assembly and stabilization. Blood, 2010. 116(22): p. 4720-30.

34. Norden, P.R., et al., Cdc42 and k-Ras Control Endothelial Tubulogenesis through Apical Membrane and Cytoskeletal Polarization: Novel Stimulatory Roles for GTPase Effectors, the Small GTPases, Rac2 and Rap1b, and Inhibitory Influence of Arhgap31 and Rasa1. PLoS One, 2016. 11(1): p. e0147758.

35. Edgar, L.T., et al., Mechanical interaction of angiogenic microvessels with the extracellular matrix. J Biomech Eng, 2014. 136(2): p. 021001.

36. Hielscher, A., et al., Fibronectin Deposition Participates in Extracellular Matrix Assembly and Vascular Morphogenesis. PLoS One, 2016. 11(1): p. e0147600.

37. Stamati, K., et al., Laminin promotes vascular network formation in $3 D$ in vitro collagen scaffolds by regulating VEGF uptake. Exp Cell Res, 2014. 327(1): p. 68-77.

38. Newman, A.C., et al., The requirement for fibroblasts in angiogenesis: fibroblast-derived matrix proteins are essential for endothelial cell lumen formation. Mol Biol Cell, 2011. 22(20): p. 3791800.

39. Samal, J., et al., Co-Culture of Human Endothelial Cells and Foreskin Fibroblasts on 3D SilkFibrin Scaffolds Supports Vascularization. Macromol Biosci, 2015. 15(10): p. 1433-46.

40. Belair, D.G., et al., Human vascular tissue models formed from human induced pluripotent stem cell derived endothelial cells. Stem Cell Rev, 2015. 11(3): p. 511-25.

41. Nishiguchi, A., et al., Effects of angiogenic factors and 3D-microenvironments on vascularization within sandwich cultures. Biomaterials, 2014. 35(17): p. 4739-48.

42. Armulik, A., G. Genove, and C. Betsholtz, Pericytes: developmental, physiological, and pathological perspectives, problems, and promises. Dev Cell, 2011. 21(2): p. 193-215.

43. Kim, J., et al., Engineering of a Biomimetic Pericyte-Covered 3D Microvascular Network. PLoS One, 2015. 10(7): p. e0133880.

44. Wang, G., et al., Origin and differentiation of vascular smooth muscle cells. J Physiol, 2015. 593(14): p. 3013-30.

45. Sacharidou, A., A.N. Stratman, and G.E. Davis, Molecular mechanisms controlling vascular lumen formation in three-dimensional extracellular matrices. Cells Tissues Organs, 2012. 195(1-2): p. 122-43. 
46. Hanjaya-Putra, D., et al., Controlled activation of morphogenesis to generate a functional human microvasculature in a synthetic matrix. Blood, 2011. 118(3): p. 804-15.

47. Shen, Y.I., et al., Hyaluronic acid hydrogel stiffness and oxygen tension affect cancer cell fate and endothelial sprouting. Biomater Sci, 2014. 2(5): p. 655-665.

48. Blatchley, M., K.M. Park, and S. Gerecht, Designer Hydrogels for Precision Control of Oxygen Tension and Mechanical Properties. J Mater Chem B Mater Biol Med, 2015. 3(40): p. 79397949.

49. Blatchley, M.R. and S. Gerecht, Acellular implantable and injectable hydrogels for vascular regeneration. Biomed Mater, 2015. 10(3): p. 034001.

50. Peters, E.B., et al., Poly(ethylene glycol) Hydrogel Scaffolds Containing Cell-Adhesive and Protease-Sensitive Peptides Support Microvessel Formation by Endothelial Progenitor Cells. Cell Mol Bioeng, 2016. 9(1): p. 38-54.

51. Nguyen, E.H., et al., Differential effects of cell adhesion, modulus and VEGFR-2 inhibition on capillary network formation in synthetic hydrogel arrays. Biomaterials, 2014. 35(7): p. 2149-61.

52. Zanotelli, M.R., et al., Stable engineered vascular networks from human induced pluripotent stem cell-derived endothelial cells cultured in synthetic hydrogels. Acta Biomater, 2016. 35: p. $32-41$. 
Figure Legends

\section{Graphical Abstract:}

Figure 1: Structural differences between pericyte-EC and vSMC-EC interactions. (A). Pericytes are characterized as being imbedded within the basement membrane of ECs in capillaries and small microvessels. (B). vSMC interact with vascular tubes with larger diameters and secrete ECM. Both cell types wrap around abluminal surface of ECs providing structural support. Fibroblasts also provide physical support to EC networks as well as necessary chemical cues.

Figure 2: Vascular networks in semi-synthetic hydrogels. (A) ECFCs forming complex vascular networks in AHA hydrogels. Taken from [44]. (B) ECFC encapsulated in hypoxic hydrogels, including stiff (gelatin-dextran) and soft (gelatin only) undergo different kinetics of vascular network formation. Taken from [46].

Figure 3: Human iPSC-derived vascular networks in 3D collagen matrix. (A) Light microscopy image and $(B)$ 3D projection of confocal image showing vascular networks formed by hiPSC-derived ECs embedded in collagen gels. Approximately 300 micron in Z direction. VECad in red; Nuclei in blue.

Figure 4: Vascular assembly kinetics. Schematic (upper panel) and corresponding light microscopy images (lower panel) showing the progression of vascular assembly of iPSC derived ECs encapsulated in collagen gels ( $\mathrm{t}=0 \mathrm{hrs})$, including vacuole formation $(\mathrm{t}=3 \mathrm{hrs})$, sprouting events $(\mathrm{t}=24 \mathrm{hrs})$, and network growth ( $\mathrm{t}=48 \mathrm{hrs})$. Graphics not drawn to scale. 
A.

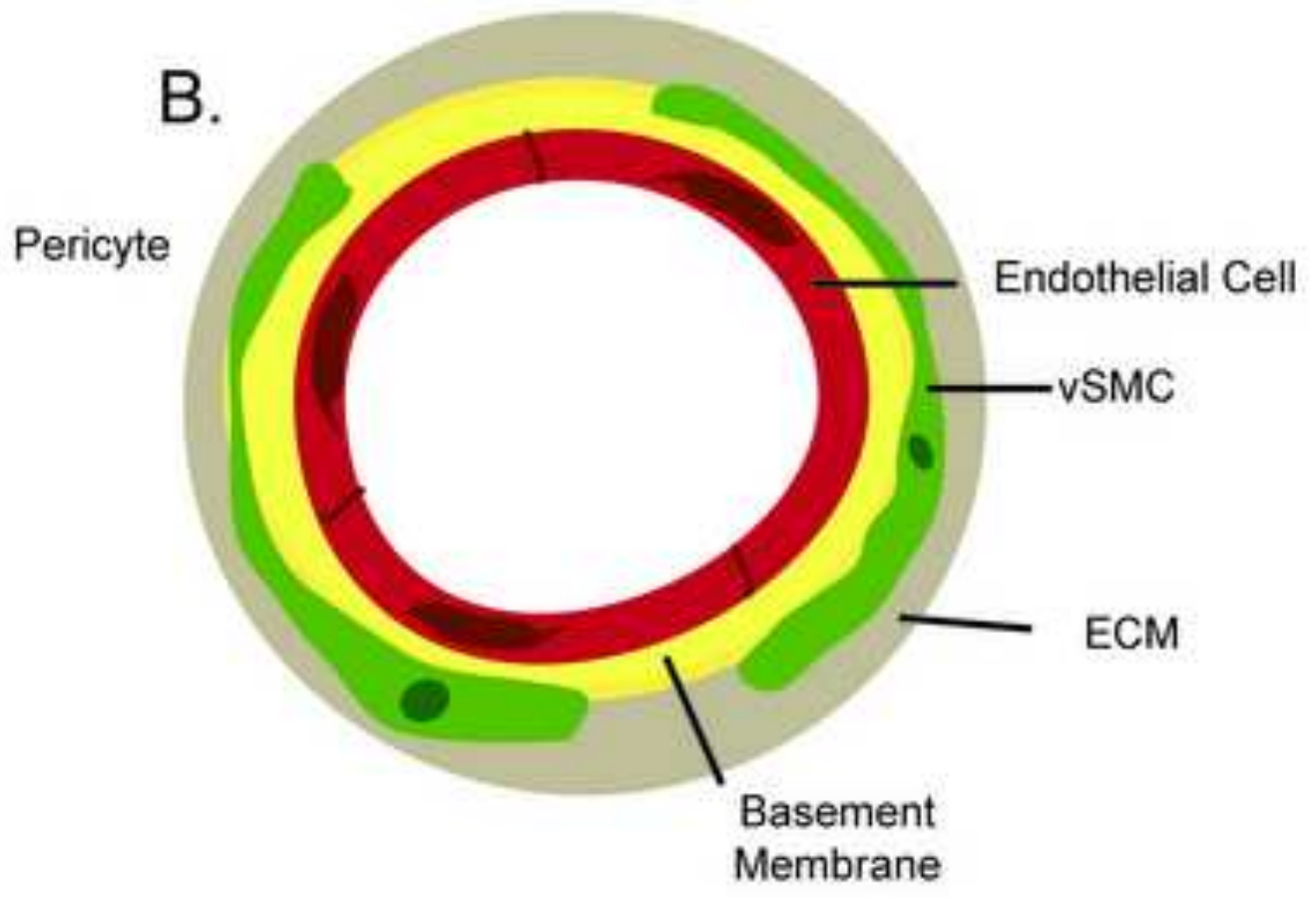
Membrane

Endothelial Cell

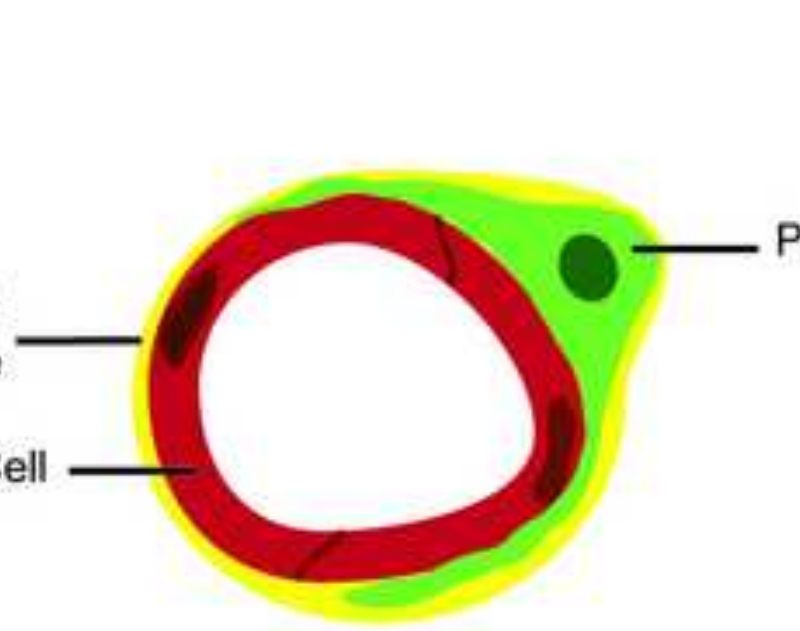

B.

Membrane 
A

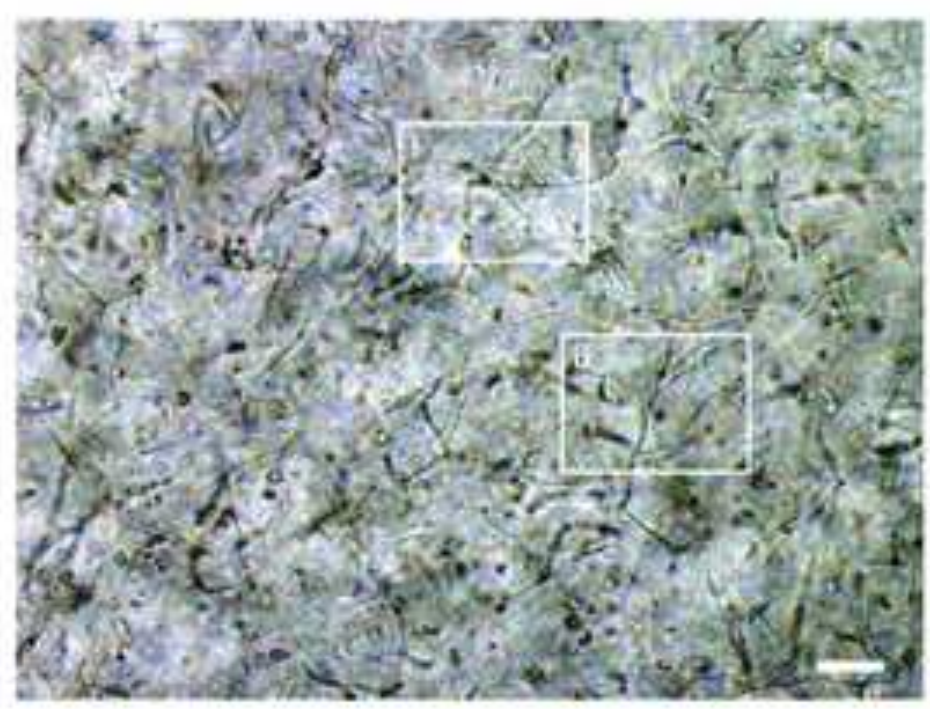

B
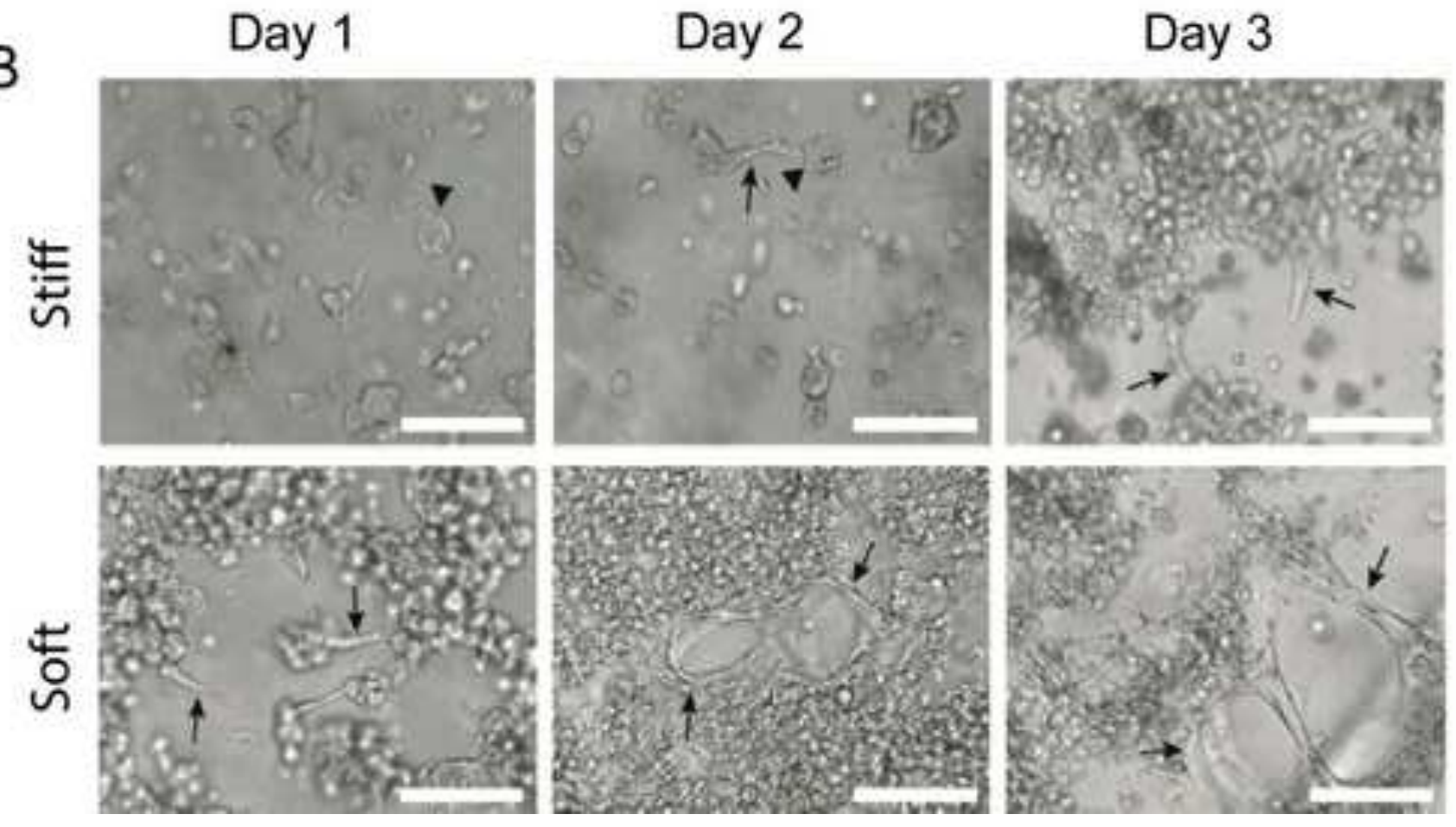

Day 2
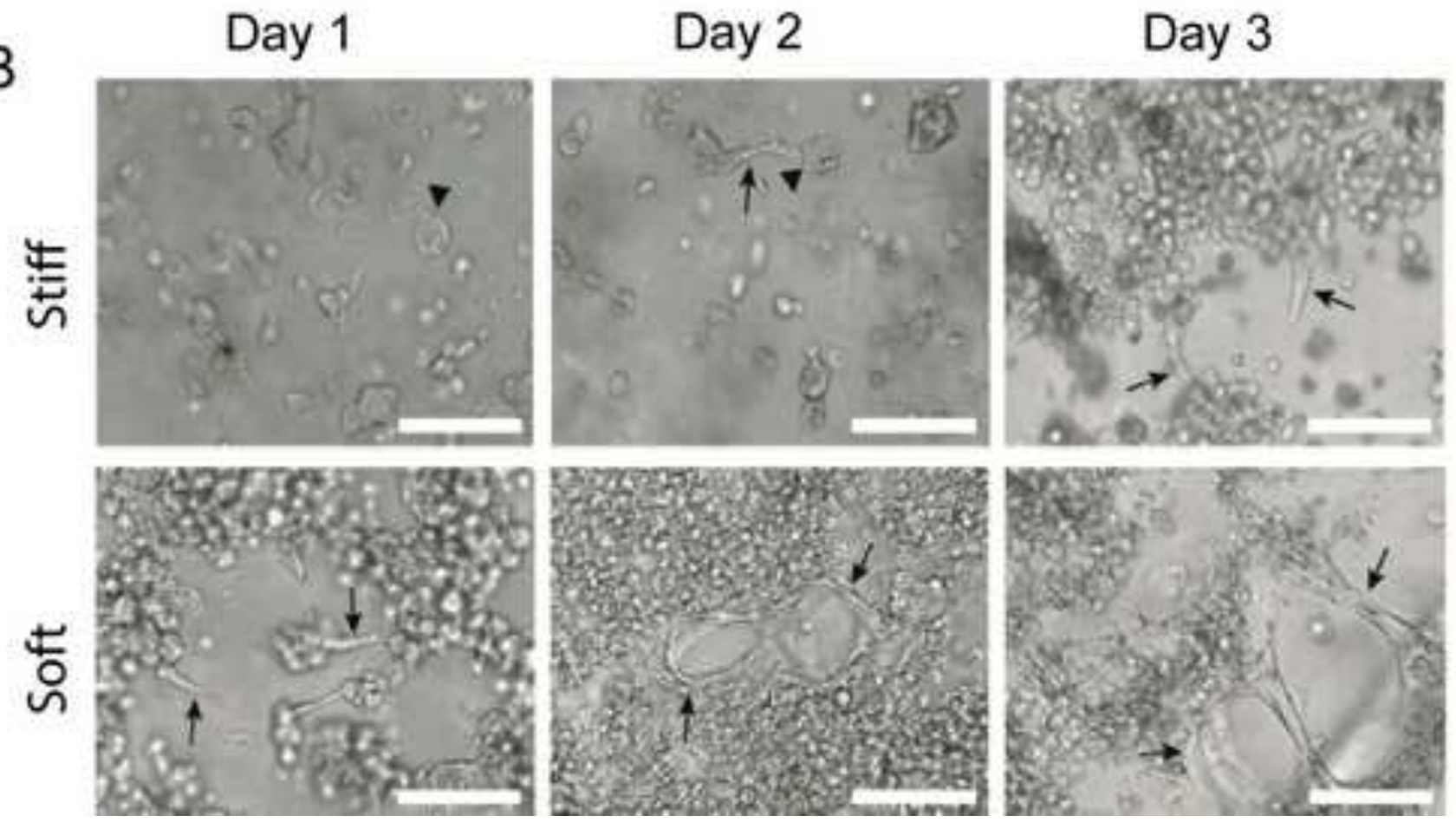

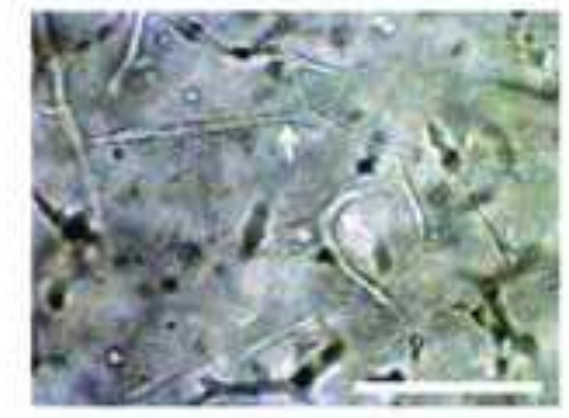

ii

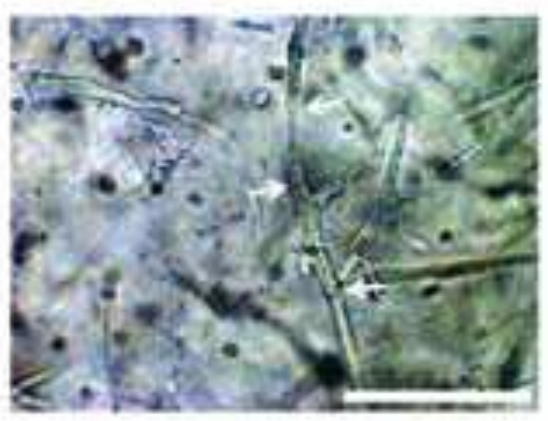

Day 3
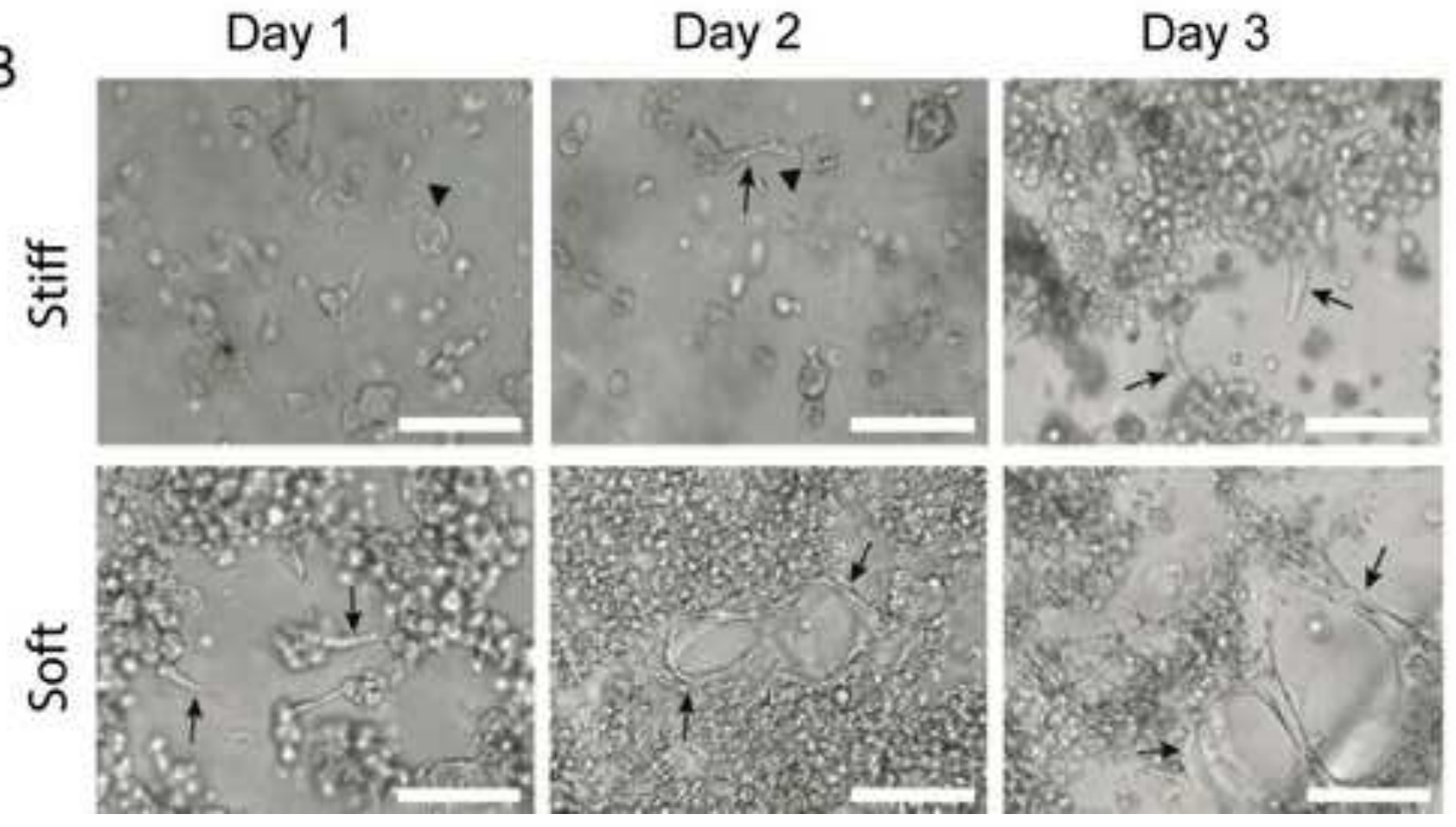
A

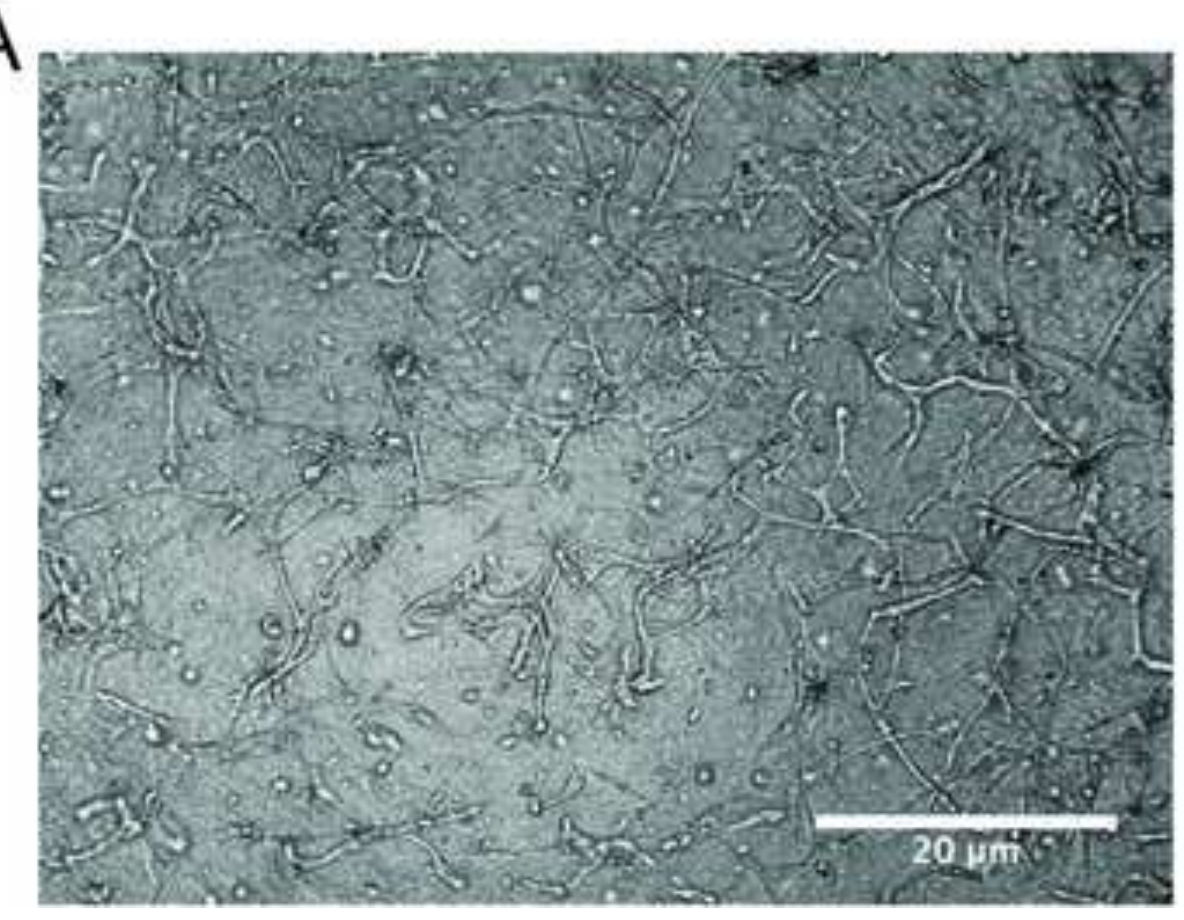

B

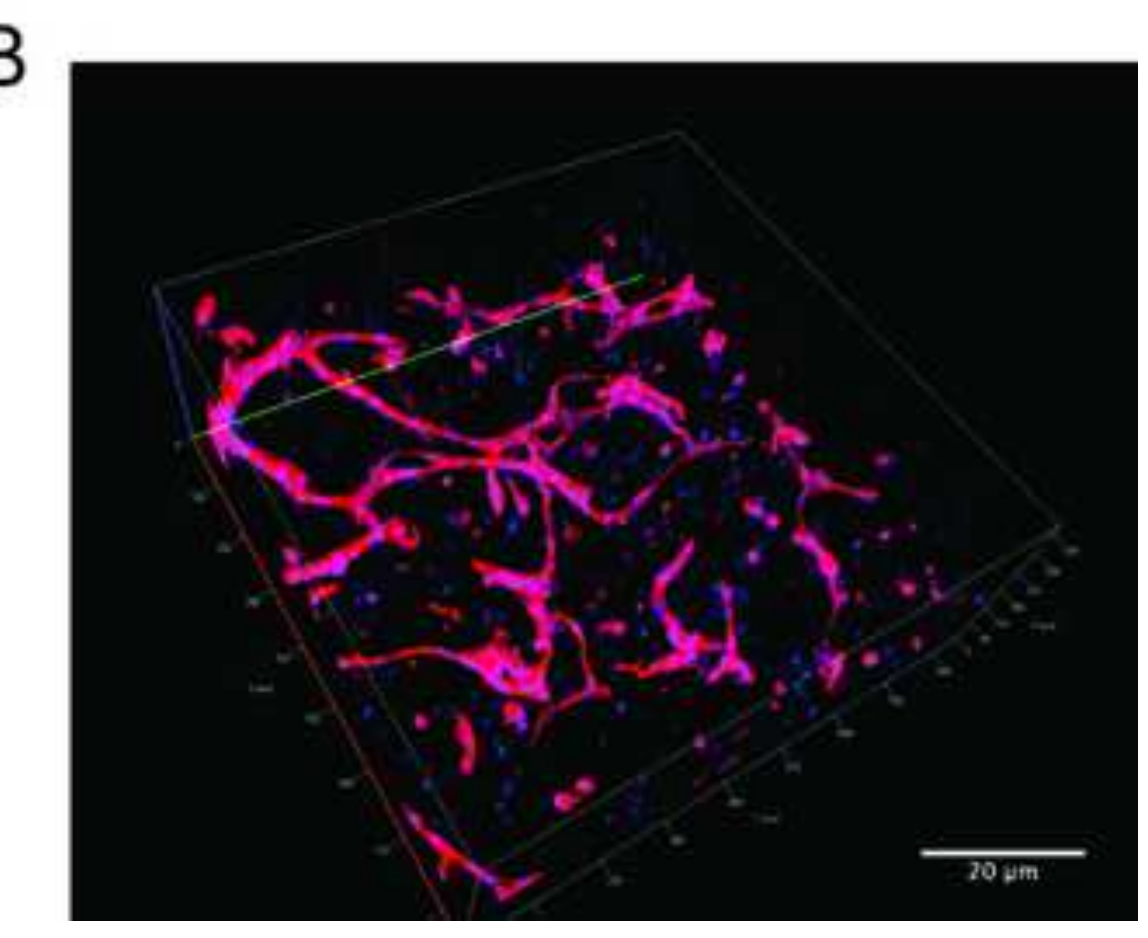

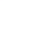



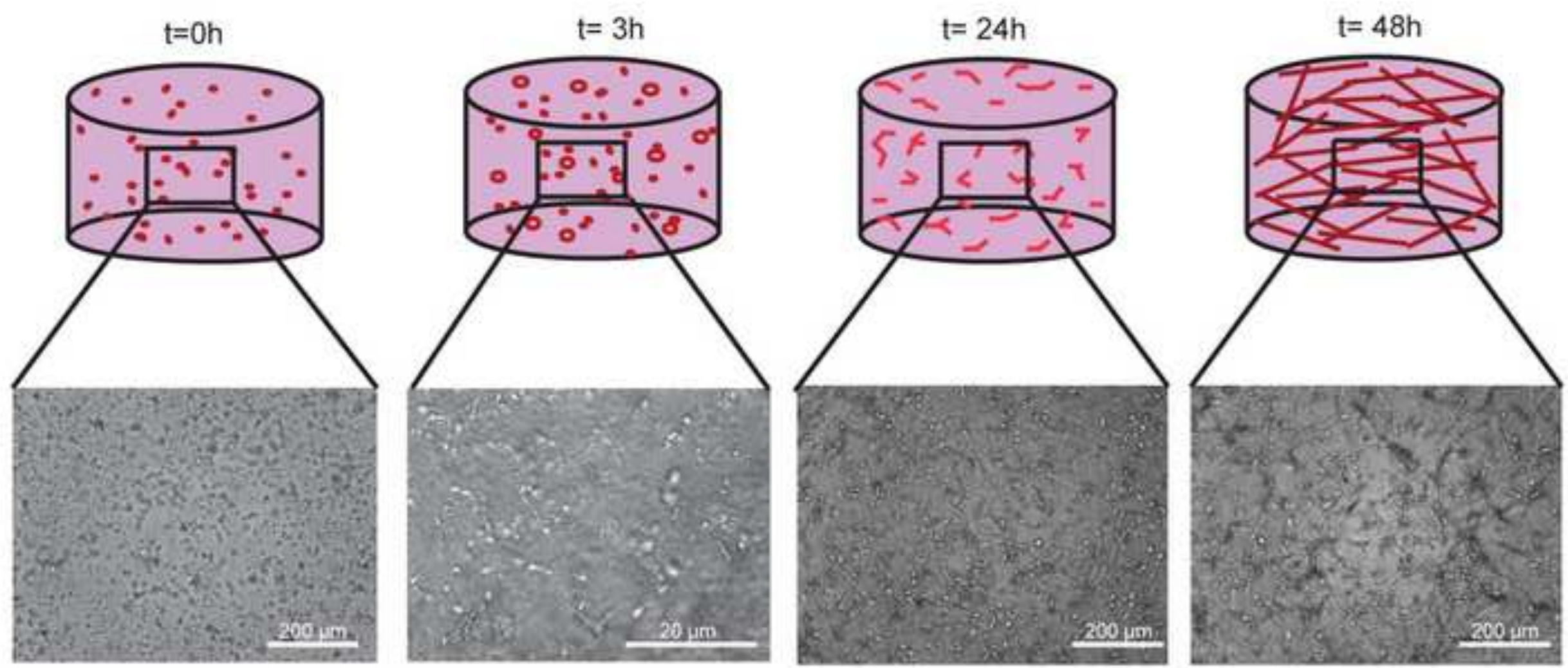
Cell Sources for Vascular Therapies

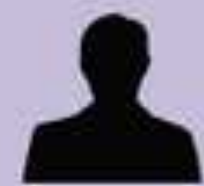

Mature Celis

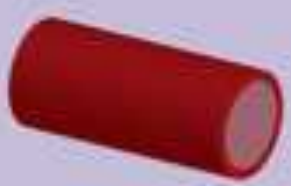

Adult Stem Cells

$$
8 \%
$$

Embryonic Stem Cells

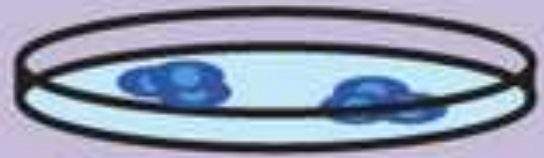

iPSC

\section{iPSC Differentiation to ECs}

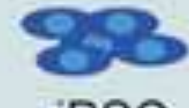

iPSC

CHIR BMP-4 Activin A

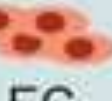

EC Isolation
FACS, MACS
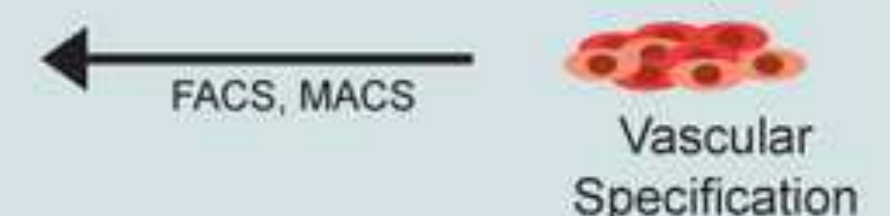

Biomaterials for Vascular Therapy

Natural Materials

collagen

fibrin

elastin

Synthetic Materials

poly (ethelyne

glycol) PEG

Semi-Synthetic Materials

acrylated hyaluronic acid

dextran based

\section{Vascular Network Assembly}

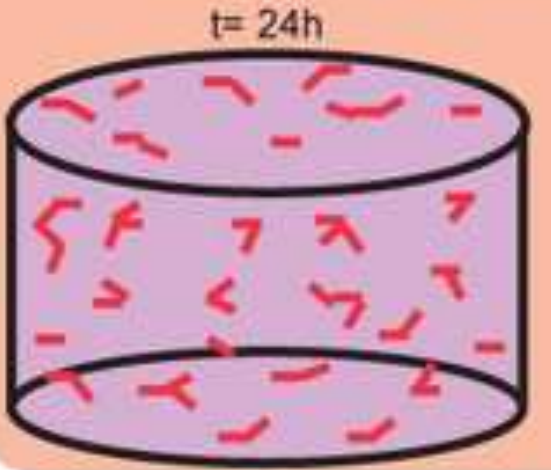

